\section{Clonidine and methylphenidate were effective for attention deficit hyperactivity disorder in children with comorbid tics}

The Tourette's Syndrome Study Group. Treatment of ADHD in children with tics. A randomized controlled trial. Neurology 2002 Feb 26;58:527-36.

\section{QUESTION: In children with attention deficit hyperactivity disorder (ADHD) and Tourette's syndrome (TS), what is the effect of clonidine (CLON), methylphenidate $(\mathrm{MPH})$, or combined CLON and MPH?}

Sources of funding:

National Institutes of

Health; Tourette

Syndrome Association.

For correspondence:

Dr R Kurlan,

University of Rochester

Medical Center,

Rochester, New York,

USA.

roger_kurlan@

urmc.rochester.edu.

Abstract and

commentary also appear in Evidence-Based

Mental Health.

\section{Design}

Randomised (allocation concealed*), blinded \{patients, clinicians, data collectors, outcome assessors, data analysts, data safety and monitoring committee, and manuscript writers ${ }^{*}+$, placebo controlled trial with 16 weeks of follow up.

\section{Setting}

11 sites in the US.

\section{Patients}

136 children between 7 and 14 years of age (mean age $10 \mathrm{y}, 85 \%$ boys) who had ADHD of any subtype and TS, chronic motor tic disorder, or chronic vocal tic disorder. Exclusion criteria included secondary tic disorder, major depression, pervasive developmental disorder, autism, mental retardation, anorexia nervosa, and bulimia. Follow up was $86 \%$.

\section{Intervention}

Children were allocated to receive CLON alone $(\mathrm{n}=34)$, MPH alone ( $n=37)$, combined CLON and MPH $(n=33)$, or placebo $(n=32)$. Weeks 1 to 4 were a dosage titration period for CLON (0.1 mg tablets or matching placebo). Children continued to receive CLON (or placebo) during weeks 5 to 8 , which was the dosage titration period for MPH ( $5 \mathrm{mg}$ capsules or matching placebo).

\section{Main outcome measures}

Severity of ADHD symptoms measured by the Conners Abbreviated Symptom Questionnaire for Teachers (ASQTeacher), tic severity measured by the Yale Global Tic Severity Scale (YGTSS), and global functioning measured by the Children's Global Assessment Scale (C-GAS).

\section{Main results}

Results are in the table. CLON (used alone or with MPH) compared with no CLON, MPH (used alone or with CLON) compared with no MPH, and combined CLON and MPH compared with placebo showed beneficial effects for ADHD symptoms as measured by the ASQ-Teacher. The greatest benefit was seen with combined CLON and MPH, which was most effective for lessening tic severity and improving global functioning, but CLON or MPH alone was also effective.

\section{Conclusion}

Clonidine or methylphenidate used alone, and especially in combination, was effective for attention deficit hyperactivity disorder in children with comorbid tics. ${ }^{3}$

*See glossary.

†nformation provided by author.
Treatment effects for clonidine (CLON), methylphenidate (MPH), combined CLON and MPH, and placebo in children with attention deficit hyperactivity disorder (ADHD) and comorbid tics at 16 weeks:

\begin{tabular}{llll} 
Outcomes & Comparisons & $\begin{array}{l}\text { Treatment effects } \\
(95 \% \mathrm{Cl})\end{array}$ & $\begin{array}{l}\text { p } \\
\text { Value }\end{array}$ \\
$\begin{array}{l}\text { ADHD (Connors Abbreviated Symptom } \\
\text { Questionnaire for Teachers) }\end{array}$ & CLON $v$ no CLON & $3.2(1.2$ to 5.2$)$ & 0.002 \\
\hline & MPH $v$ no MPH & $3.2(1.1$ to 5.2$)$ & 0.003 \\
\hline & CLON + MPH $v$ placebo & $6.3(2.8$ to 9.8$)$ & $<0.0001$ \\
\hline Tic severity (Yale Global Tic Severity Scale) & CLON $v$ no CLON & $6.2(1.1$ to 11.3$)$ & 0.02 \\
\hline & MPH $v$ no MPH & $4.8(-0.4$ to 9.9$)$ & $0.07 \S$ \\
\hline & CLON + MPH $v$ placebo & $11.0(2.1$ to 19.8$)$ & 0.003 \\
\hline $\begin{array}{c}\text { Global functioning (Children's Global } \\
\text { Assessment Scale) }\end{array}$ & CLON $v$ no CLON & $6.9(2.7$ to 11.1$)$ & 0.002 \\
\hline & MPH $v$ no MPH & $7.7(3.4$ to 11.9$)$ & 0.0005 \\
\hline & CLON + MPH $v$ placebo & $14.5(7.2$ to 21.9$)$ & $<0.0001$ \\
\hline
\end{tabular}

tPositive values show a beneficial effect of the medication.

$\S$ Not significant.

\section{COMMENTARY}

The well designed trial by the Tourette's Syndrome Study Group addresses various questions related to treating $\mathrm{ADHD}$ in patients with tics. In terms of treatment effectiveness, CLON, MPH, and combined CLON and MPH were all more effective than was placebo for treating symptoms of $\mathrm{ADHD}$, and the greatest treatment effect was seen with combined CLON and MPH. The differential effects of CLON and MPH on symptoms of ADHD were as follows: MPH provides a broader therapeutic coverage of ADHD symptoms; MPH is superior to CLON in treating cognitive symptoms of inattention; CLON provides complementary benefit for treating hyperactive and impulsive behaviours; and combined CLON and MPH has the greatest effect on global functioning. With respect to adverse effects, combined CLON and MPH was most effective for lessening tic severity, but more patients receiving MPH alone reported tics as a reason for limiting further increases in their MPH dose. However, the reported frequency of tic worsening was the same across all treatments, which is supportive of other studies on MPH and tics. ${ }^{1}$ CLON alone or combined with MPH was not associated with adverse cardiac effects, but the study excluded patients with known cardiac problems. Thus, the safety of combined CLON and MPH in this group was not addressed.

The study supports the effectiveness and safety of MPH, CLON, or the 2 drugs combined for treating symptoms of ADHD in children with tics. Tics were not substantially worse in the treatment group than in the placebo group; however, the duration of the trial (16 wks) is insufficient to determine the long term safety of MPH for tics. Furthermore, the study does not address the relation of MPH dose and the emergence of tics, which is an important clinical issue. ${ }^{2}$ This study agrees with other reports on the safety of using MPH and CLON. However, careful history, examination, and monitoring are still warranted. ${ }^{3} 4$

J Goldberg, MB, FRCPC McMaster University Hamilton, Ontario, Canada

Gadow KD, Sverd J, Sprafkin J, et al. Arch Gen Psychiatry 1999;56:330-6. Castellanos FX, Giedd JN, Elia J, et al. J Am Acad Child Adolesc Psychiatry 1997;36: 589-96. Popper C. J Child Adolesc Psychopharmacol 1995;5:157-66.

Swanson JM, Flockhart D, Udrea D, et al.J Adolesc Psychopharmacol 1995;5:301-4. 